\title{
Gambling Increases Self-Control Strength in Problem Gamblers
}

\author{
Anne E. Bergen • Ian R. Newby-Clark • Andrea Brown
}

Published online: 22 November 2012

(C) Springer Science+Business Media New York 2012

\begin{abstract}
In two studies it is demonstrated that, in the short-term, slot machine gambling increases self-control strength in problem gamblers. In Study $1(N=180)$, participants were randomly assigned to either play slot machines or engage in a control task (word anagrams) for $15 \mathrm{~min}$. Subsequent self-control strength was measured via persistence on an impossible tracing task. Replicating Bergen et al. (J Gambl Stud, doi:10.1007/s10899011-9274-9, 2011), control condition participants categorized as problem gamblers persisted for less time than did lower gambling risk participants. However, in the slot machine condition, there were no significant differences in persistence amongst participants as a function of their gambling classification. Moreover, problem gambling participants in the slot machine condition persisted at the impossible tracing task longer than did problem gambling participants in the control condition. Study $2(N=209)$ systematically replicated Study 1. All participants initially completed two tasks known to deplete selfcontrol strength and a different control condition (math problems) was used. Study 2 results were highly similar to those of Study 1 . The results of the studies have implications for the helping professions. Specifically, helping professionals should be aware that problem gamblers might seek out gambling as a means of increasing self-control strength.
\end{abstract}

Keywords Self-control $\cdot$ Problem gambling $\cdot$ Slot machines

Lack of self-control is believed to be a key cause of problem gambling behaviour (Baumeister et al. 1994; Blaszczynski and Nower 2002; O'Connor and Dickerson 2003). Blazynski and Nower (2002, p. 488) point out that “... the defining feature of a problem gambler is not only the emergence of negative consequences but also the presence of a subjective sense of impaired control." Along with activities like smoking, drinking, and impulsive spending, problem gambling involves a perceived inability to exert control over an unwanted behaviour (Baumeister et al. 1994). Of importance, evidence is mounting that problem gamblers' low self-control is not limited to the domain of gambling. Using

A. E. Bergen $(\bowtie) \cdot$ I. R. Newby-Clark · A. Brown

University of Guelph, 50 Stone Road East, Guelph, ON N1G 2W1, Canada

e-mail: bergena@uoguelph.ca 
self-report and behavioural measures, Bergen et al. (2011) demonstrated that problem gamblers evidence lower trait self-control than do lower-risk gamblers.

Bergen et al. (2011) used the strength model of self-control (Baumeister et al. 2007) to operationalize self-control. The strength model of self-control posits that self-control strength is a limited resource that is used in self-regulatory acts of all kinds. Self-control strength varies across persons. It also varies within persons as a function of self-control exertion (e.g., Baumeister et al. 1998, 2007). Diverse regulatory actions, including control over emotions, attention, impulses, and choices, are fueled by a common reservoir of selfcontrol (for a meta-analysis see Hagger et al. 2010). Each of these regulatory actions requires executive control (i.e., volitional and deliberate control over behaviour).

Bergen et al.'s finding of low trait self-control in problem gamblers is worrying because self-control strength is a general strength for diverse self-regulatory acts. A chronic lack of self-control amongst problem gamblers means that everyday self-regulatory acts are less likely to be completed and that the seeking of immediate short-term gratification will occur often and at the expense of more salubrious behaviours. Indeed, there is a substantial literature to suggest that such is the case. Compared to people with less severe gambling pathology, problem gamblers use less successful problem solving orientations (Clarke 2004; Ladouceur et al. 1997), show deficits in general decision making (Goudriaan et al. 2005), tend to have lower grades and engage in more delinquent behaviour (Ladouceur et al. 1999), and are more likely to evidence comorbid substance abuse problems (Baron and Dickerson 1999; Ladouceur et al. 1997, 1999; Nower et al. 2004).

Although changing people's dispositional (i.e., trait) self-control strength can be difficult, there are many ways to temporarily increase self-control strength. Getting some sleep (Muraven et al. 2005), listening to soothing music (Tyler and Burns 2008), receiving a surprising gift, watching a humorous movie (Tice et al. 2007), receiving a boost in positive affect (Shmueli and Prochaska 2009), or just taking a break (Neri et al. 2002; Tucker 2003; Tyler and Burns 2008), can all increase self-control strength in the short-term (i.e., state self-control).

Activities that result in the short-term increase of self-control require little, if any, selfcontrol to complete. Presumably, if self-control strength is to be increased it must not be actively in demand. Also, such activities tend to be enjoyable ones, granting people a respite that can be energizing (see Thayer et al. 1994; Tice et al. 2007). We suggest that, for problem gamblers in particular, gambling has this same character.

Gambling often does not require a great deal of self-control (a feature that may have attracted people with low self-control to gambling in the first place). Especially among problem gamblers, gambling is an enjoyable activity (Gupta and Deverensky 1998; Neighbors et al. 2002) an escape from daily problems (i.e., respite), or a form of relaxation (Clarke 2008; Gupta and Deverensky 1998) It would therefore appear that gambling can be a low self-control activity that is, especially for problem gamblers, enjoyable, and provides needed respite from everyday hassles and boredom. It is thus possible that problem gamblers will increase their self-control strength, albeit temporarily, by gambling.

Such a finding would have important implications for researchers and practitioners alike because it suggests another reason why problem gamblers gamble. That is, beyond the desire to escape boredom per se (Nower et al. 2004), and beyond cognitive distortions (Johansson et al. 2009), it could be that problem gamblers gamble because it results in a short-term payoff of greater self-control. Interventions would thus need to emphasize to problem gamblers that there are less harmful, and often beneficial, ways to improve selfcontrol in the short-term and the long-term. 
'Games of chance' vary in the degree of self-control that must be exerted during play. In poker, for example, players must decide on how much money to bet and whether or not to take a card, controlling their emotional expressions all the while. There is now a good deal of evidence that making decisions and controlling facial expressions are activities that decrease self-control strength (Vohs et al. 2008; Vohs and Heatherton 2000). Other kinds of gambling involve some active deliberation and decision-making, such as blackjack (whether or not to 'stick' or 'hit'), whereas others involve little to no decision-making (e.g., scratching a lottery ticket or pulling the lever of a slot machine). If gambling can increase self-control strength, it is most likely to do so when gambling activities exert little to no demand on self-control resources. In the current research, we thus elected to focus on slot machine play, a highly popular form of gambling to which problem gamblers are particularly attracted (Fisher and Griffiths 1995).

In two studies, we test for the possibility that problem gamblers' self-control strength is greater after slot machine gambling and we obtain evidence that suggests this increase in self-control after gambling is specific to problem gamblers. In Study 1 we show that when problem gamblers do not gamble, they evidence a relatively lower amount of self-control than do non-problem gamblers (replicating Bergen et al. 2011). When problem gamblers gamble, though, they show a significant increase in self-control, thus reducing their selfcontrol discrepancy with non-problem gamblers. In Study 2, we expand on these findings, demonstrating the same interaction pattern after participants first engage in tasks known to deplete self-control resources.

\section{Study 1}

Method

\section{Participants}

A total of 180 participants (91 female) were recruited for a study on gambling and cognition. Fifty-three participants (19 female) were recruited from the first year psychology participant pool at the University of the Guelph (Ontario, Canada). Another 127 participants were recruited from the community ( 72 female) by various means (posters, email, word of mouth and a gambling researchers' database). Student participants were compensated with course credit; community participants were remunerated with \$25 CAD.

\section{Procedures}

Upon arrival at the lab, participants gave written informed consent. The lab environment was a room containing several slot machines.

Self-Control Depletion Community participants then completed the Problem Gambling Severity Index (PGSI; Ferris and Wynne 2001). The PGSI is a nine-item scale that distinguishes four subtypes of gamblers: non-gamblers, low-risk gamblers, moderate-risk gamblers, and problem gamblers. The PGSI was designed for use in general populations, and has demonstrated adequate internal consistency, test-retest reliability, and validity (Ferris and Wynne 2001; Newby-Clark and Brown 2008). Student participants completed the PGSI in computerized mass testing at the beginning of the academic term. 
Gambling Manipulation Experimental condition was determined through random assignment to either the slot machine or word game condition. Participants in the slot machine condition were provided with tokens to play a slot machine for 15 min. Participants in the word game condition were occupied with a word game (finding smaller words in larger words). After 15 min had elapsed, participants' self-control strength was assessed using a behavioural measure: persistence on an impossible tracing task.

Self-Control Strength This reliable and well-validated measure of self-control was devised by Baumeister et al. (1998) and has been used successfully by many self-control researchers (see Hagger et al. 2010). Specifically, participants were given tracing puzzles that, unbeknownst to them, were unsolvable. The puzzles required participants to trace geometric shapes without retracing any lines and without lifting pencil from paper. Multiple pieces of paper were provided to allow participants to make repeated attempts at each shape. Participants were initially given two solvable practice shapes, with the experimenter present to answer any questions. After the practice period, the experimenter gave participants the two impossible tracing tasks, one after the other. Participants were informed that they could give up working on a particular shape at any time. Thus, the main dependent measure was total persistence time, with longer persistence at this frustrating task representing greater self-control. Following Baumeister et al. (1998), 30 min was set as the maximum time each participant could work at the tracing task. At the end of the study, participants were debriefed fully and compensated for their time.

\section{Results}

Participants who scored zero on the PGSI ( $n=43 ; n=12$ males) were categorized as non-gamblers. There were 45 participants ( $n=20$ males) categorized as low risk gamblers (PGSI scores of one and two), 70 participants $(n=37$ males) categorized as moderate risk gamblers (scores between three and seven, inclusive), and 22 participants ( $n=20$ males) categorized as problem gamblers (scores of eight or higher).

It would appear that problem gambling severity was related to gender, such that higherrisk gamblers were more likely to be male and lower-risk gamblers were more likely to be female. A Spearman correlation coefficient confirmed the significance of the relation, $r_{S}$ $(178)=-.33, p<.001$. This finding is consistent with previous research showing that male gender is a risk factor for problem gambling (Johansson et al. 2009), especially in young adults and adolescents (Welte et al. 2008). Due to the low number of female problem gamblers $(n=2)$, analyses by gender could not be conducted.

Following Baumeister et al. (1998), the total amount of time spent on the two unsolvable puzzles was calculated for each participant. As the data were not normally distributed, a base ten logarithmic transformation was applied to the data to mitigate kurtosis. A between-groups 2 (condition) $\times 4$ (gambling severity category) ANOVA revealed a marginally significant interaction, $F(3,172)=2.35, p=.07, \eta_{p}^{2}=.04$. Untransformed means are displayed in Fig. 1 to aid interpretation.

The interaction was investigated using simple effects analyses, which allowed us to separately examine the effect of gambling severity within the word game and slot machine conditions. Group differences within experimental conditions were further investigated using Fisher's Least Squares Difference (LSD) multiple comparisons, comparing average scores of PGSI gambling risk groups against one another. The simple effect of gambling severity within the word games condition was significant, $F(3,172)=2.72, p=.05$, 


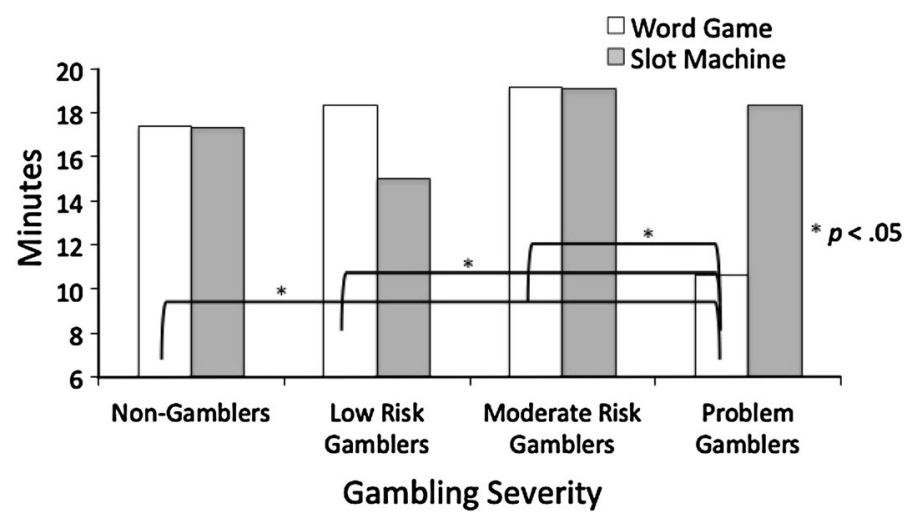

Fig. 1 Problem gamblers show self-control deficits in control condition, but not after gambling on slot machine (Study 1)

$\eta_{p}^{2}=.05$, and Fisher's LSD multiple comparisons revealed significant differences between problem gamblers and each of the other three gambling severity categories. In the slot machine condition, by contrast, there was no simple effect of gambling severity, $F(3$, $172)=1.09, p=.35, \eta_{p}^{2}=.02$. Simple effects analyses also revealed a significant effect of condition only for the highest level of problem gambling severity, $F(1,172)=4.62$, $p=.03, \eta_{p}^{2}=.03$ (all other $F$ 's $<1.97, p$ 's $>.16$ ), with problem gamblers evidencing more persistence in the slot machine condition than they did in the word game condition.

\section{Study 2}

Study 1 provided compelling initial evidence that problem gamblers' self-control strength increases after gambling. As expected, problem gamblers in the word game (i.e., control) condition evidenced less persistence on the unsolvable puzzles than did participants with less severe gambling pathology. This pattern of effects replicates Bergen et al.'s (2011) findings in their two studies. In the slot machine (i.e., gambling) condition, the difference between problem gamblers and other participants decreased substantially; so much so that, in fact, problem gamblers in the gambling condition persisted for longer at the unsolvable puzzles than did problem gamblers in the control condition.

The novelty of our results- to our knowledge, this is the first time that such an effect has been obtained-and the marginal significance of the interaction compelled us to undertake a systematic replication.

In Study 2 we retained essential elements of Study 1, but strengthened our approach in a number of ways. First, we opted to recruit solely from the community so that we could obtain a heterogeneous sample using one means of compensation for participation (in case method of compensation impacted motivation to participate). Second, although it was the case that the control condition results in Study 1 were highly similar to those of Bergen et al. (2011), confidence in our findings would be higher if we contrasted our gambling condition with a control condition of a different kind. We thus opted to give Study 2 control condition participants moderately difficult math multiplication problems.

Third, and most important, we began Study 2 by requiring all participants to complete two self-control depletion tasks before they were randomly assigned to the experimental 
condition. We did this to rule out the possibility that non-problem gamblers were performing at their self-control strength 'ceiling' in Study 1 and were therefore unable to show any benefit that accrued to them by gambling. The two self-control activities were based on established methods designed to deplete self-control strength (e.g., Baumeister et al. 1998, p. 1258; Muraven et al. 1998; Muraven and Slessareva 2003).

\section{Method}

\section{Participants}

Participants were 220 people recruited from a database containing the contact information of community members who expressed interest in regularly participating in gambling research. Participants were also recruited via newspaper and circular advertising, online advertising, and word-of-mouth via previous participants. All participants were remunerated with a movie pass valued at $\$ 10 \mathrm{CAD}$. Of the 220 people who participated in the study, four had incomplete data or accidentally participated in the study more than once and another seven did not follow experimental instructions. Of the remaining 209 participants, 115 were female. Of the 207 participants who reported their age, ages ranged from 18 to 87 years $(M=43, \mathrm{SD}=19)$.

\section{Procedure}

The lab environment was a room containing several slot machines. Upon arrival at the lab, participants were told that they would be engaging in a series of activities designed to test their self-control performance and that they would be filling out a questionnaire to assess their gambling behaviour. They then gave informed consent.

Participants were tested in groups of up to four and participants in the same session were in the same experimental condition. Upon entering the lab, participants completed a consent form explaining the nature of the study. Next, as in Study 1, all participants completed the PGSI as a measure of problem gambling severity (Ferris and Wynne 2001).

Self-Control Depletion Depletion was accomplished with two activities known to quickly deplete self-control: a cognitive processing task (anagram solving) and a thought control activity. Meta-analysis shows that both tasks have a moderate effect size (Hagger et al. 2010). Two tasks were used in order to increase the likelihood of self-control depletion. In the anagrams task, participants were first instructed to solve moderately challenging anagrams for $5 \mathrm{~min}$. Participants were told to "Work on the anagrams one at a time in order, making sure you complete an anagram before moving onto the next one." After working on the anagrams for $5 \mathrm{~min}$, participants were instructed to list their thoughts on a piece of paper while suppressing all thoughts of a white bear (indicating on the paper every time their thoughts turned to white bears). Thought suppression has been shown to deplete self-control strength (e.g., Baumeister et al. 1998, p. 1258; Muraven et al. 1998; Muraven and Slessareva 2003). The thought suppression depletion phase continued for $5 \mathrm{~min}$, after which participants completed the gambling manipulation.

Gambling Manipulation Participants were randomly assigned to either the control condition or the gambling condition. Participants in the control condition answered moderately difficult multiplication problems for $15 \mathrm{~min}$ and participants in the gambling condition 
gambled on slot machines for $15 \mathrm{~min}$. In order to increase the ecological validity of the gambling task, participants were told that if they ended up with more tokens than they started with after gambling, they would receive twice the value in movie passes for their participation (\$20 instead of \$10).

Self-Control Strength As in Study 1, self-control strength was assessed as persistence on an impossible tracing task (Baumeister et al. 1998; Muraven and Slessareva 2003; NewbyClark and Brown 2008).

Results

Participants who scored zero on the CGPI ( $n=53 ; n=20$ male) were categorized as nongamblers. There were 59 participants $(n=22$ males) categorized as low risk gamblers (CGPI scores of one and two), 74 participants $(n=39$ males) categorized as moderate risk gamblers (scores between three and seven, inclusive), and 23 participants ( $n=13$ males) categorized as problem gamblers (scores of eight or higher). As in Study 1, problem gambling severity was related to gender, $r_{S}(207)=-.15, p<.05$, such that higher-risk gamblers were more likely to be male and lower-risk gamblers were more likely to be female. Due to the low number of problem gamblers of each gender, analyses by gender could not be conducted.

Following Baumeister et al. (1998), the total amount of time spent on the two unsolvable puzzles was calculated for each participant and a base ten logarithmic transformation was applied to the data to mitigate a kurtosis problem. Untransformed means are displayed in Fig. 2 to aid interpretation. A between-groups 2 (condition) $\times 4$ (gambling severity category) ANOVA revealed a significant interaction, $F(3,201)=2.98, p=.03$, $\eta_{p}^{2}=.04$. We next conducted planned simple effects analyses to separately examine the effect of gambling severity within the word game and slot machine conditions. Group differences within experimental conditions were further investigated using Fisher's LSD multiple comparisons, comparing average scores of PGSI gambling risk groups against one another. The simple effect of gambling severity within the word game condition was significant, $F(3,201)=2.87, p=.04, \eta_{p}^{2}=.04$, and Fisher's LSD multiple comparisons revealed significant differences between problem gamblers and the other three gambling severity categories ( $p=.08$ for the comparison with low risk gamblers). In the slot machine condition, by contrast, there was no simple effect of gambling severity, $F(3$, $201)=1.67, p=.18, \eta_{p}^{2}=.02$. Simple effects analyses also revealed a significant effect of condition only for the highest level of problem gambling severity, $F(1,201)=5.72$, $p=.02, \eta_{p}^{2}=.03$, (all other $F^{\prime}$ s $<1.95, p$ 's $>.16$ ), with problem gamblers evidencing more persistence in the slot machine condition than they did in the math problems condition.

\section{Cross-Study Analysis}

Viewed in isolation, the results of Study 1 are not entirely persuasive, partly because of the novelty of the effect we obtained, but also because it is entirely possible that participants who were not problem gamblers may have been performing at 'ceiling' in the control condition and were therefore unable to show any benefit to self-control strength by gambling. In Study 2, then, we used two well-established techniques to deplete self-control strength before randomly assigning participants to condition. We acknowledge that 


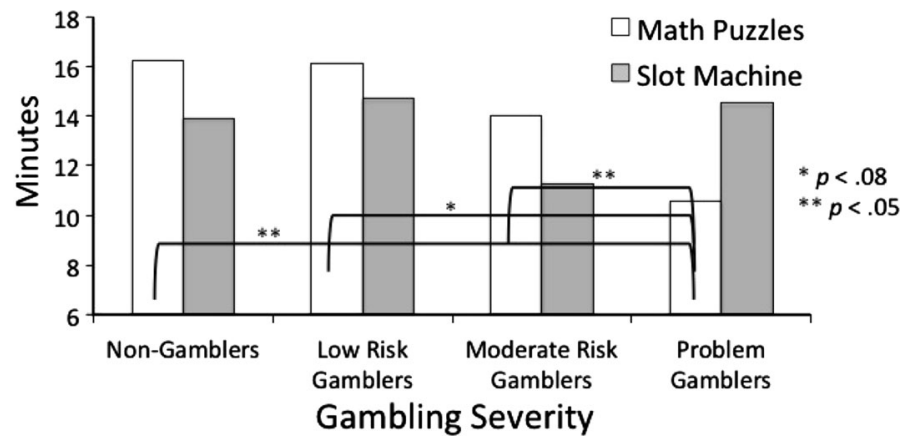

Fig. 2 Problem gamblers show self-control deficits in control condition, but not after gambling on slot machine (Study 2)

introducing a depletion/no depletion factor into our Study 2 experimental design would have been ideal to demonstrate that the depletion tasks were effective. Although it is only suggestive at best, we conducted a cross-study analysis involving participants in the control conditions. A 2 (study) $\times 4$ (gambling severity) ANOVA on persistence at the impossible tracing task revealed a strong main effect of study, $F(1,184)=9.88, p=.002, \eta_{p}^{2}=.05$, such that participants in Study 1 persisted for longer $(M=17.5 \mathrm{~min} ; \mathrm{SD}=8.17)$ than did participants in Study $2(M=14.7 \mathrm{~min}$; SD = 9.50). As might be expected, there was also a strong main effect for gambling severity, $F(3,184)=5.08, p=.002, \eta_{p}^{2}=.08$ and no interaction $(F<1)$. It would thus appear that Study 2 participants' self-control strength was depleted and there existed the potential for slot machine gambling to increase selfcontrol strength amongst Study 2 participants lower in gambling severity.

\section{General Discussion}

In two studies, we obtained two important findings. First, in a further replication of Bergen et al. (2011), we confirmed that problem gamblers exhibit less self-control strength than do people with less severe gambling problems. Specifically, in both control conditions, problem gamblers persisted at the impossible tracing task for significantly less time than did participants at lower risk for gambling problems. Second, and of greater importance, we found that slot machine gambling increases self-control strength in problem gamblers. Indeed, the now four-times replicated effect of problem gamblers being lower in selfcontrol was not in evidence in the slot machine conditions and there was a significant increase in self-control strength for problem gamblers in the slot machine conditions compared to the control conditions. There was no evidence of such an effect for participants in lower gambling severity categories. In addition, our cross-study analysis suggests that previous self-control depletion may amplify the restorative effects of gambling for problem gamblers.

In an earlier paper (Bergen et al. 2011) we demonstrated that problem gamblers are comparatively low in trait self-control, a finding that we twice-replicated here. Being chronically low in self-control strength is by no means a trifling matter. Self-control strength is required for effective self-regulation in day-to-day life. People low in selfcontrol strength will show deficits in many everyday tasks, including regulation of diet and exercise, finances and spending, and control over emotional responses (Baumeister et al. 
1994; Vohs et al. 2011; Vohs and Heatherton 2000). To the extent that any behaviour or set of behaviours increases self-control strength it should be positively reinforcing and approached. Our data point to the distinct possibility that problem gamblers will gamble not only because their self-control is low but because they wish to increase it. Future research will need to address this possibility.

Our results are limited by the fact the slot machines were played in a research lab. It may be that the current findings do not generalize to actual casino environments, or slot machine games played with real money, and the current findings should be replicated in settings with higher external validity. In addition, we did not assess all demographic variables that could have impacted self-control and problem gambling, and therefore the possibility remains that gambling impacts self-control strength differently across such factors as and education level and socioeconomic status. ${ }^{1}$ Future research should examine whether demographic differences influence the interaction between gambling and selfcontrol strength.

The improved understanding of problem gamblers that our two studies bring also has implications for intervention. Specifically, beyond the excitement, respite, and escape from the ordinary that problem gamblers often report as motivation, it is important for helping professionals to realize that gambling brings with it an additional benefit for problem gamblers and that this benefit will need to be achieved by another means. Thus, beyond improving short-term self-control strength and the benefits that would accrue from such remediation (see Bergen et al. 2011), helping professionals need to work with clients to identify behaviours and activities that require little to no self-control, and provide craved relief from boredom.

Acknowledgments This project was funded by a grant from the Ontario Problem Gambling Research Centre (www.gamblingresearch.org). Many thanks to Dr. Karen Finlay at the University of Guelph for the use of her database of community gambling participants.

\section{References}

Baron, E., \& Dickerson, M. (1999). Alcohol consumption and self-control of gambling behaviour. Journal of Gambling Studies, 15, 3-15.

Baumeister, R. F., Bratslavsky, E., Muraven, M., \& Tice, D. M. (1998). Ego depletion: Is the active self a limited resource? Journal of Personality and Social Psychology, 74, 1252-1265.

Baumeister, R. F., Heatherton, T. F., \& Tice, D. M. (1994). Losing control: How and why people fail at selfregulation. New York: Academic Press.

Baumeister, R. F., Vohs, K. D., \& Tice, D. M. (2007). The strength model of self-control. Current Directions in Psychological Science, 16, 351-355.

Bergen, A. E., Newby-Clark, I. R., \& Brown, A. (2011). Low trait self-control in problem gamblers: Evidence from self-report and behavioral measures. Journal of Gambling Studies,. doi:10.1007/ s10899-011-9274-9.

Blaszczynski, A., \& Nower, L. (2002). A pathways model of problem and pathological gambling. Addiction, 97, 487-499.

Clarke, D. (2004). Impulsiveness, locus of control, motivation and problem gambling. Journal of Gambling Studies, 20, 319-345. doi:10.1007/s10899-004-4578-7.

Clarke, D. (2008). Older adults' gambling motivation and problem gambling: A comparative study. Journal of Gambling Studies, 24, 175-192. doi:10.1007/s10899-008-9090-z.

Ferris, J., \& Wynne, H. (2001). The Canadian Problem Gambling Index. Ottawa, ON: Canadian Centre on Substance Abuse.

\footnotetext{
${ }^{1}$ In the current research, there was no significant relationship between age or gender and self-control strength, controlling for problem gambling severity (all $p$ 's $>.05$ ).
} 
Fisher, S., \& Griffiths, M. (1995). Current trends in slot machine gambling: Research and policy issues. Journal of Gambling Studies, 11, 239-247.

Goudriaan, A. E., Oosterlaan, J., de Beurs, E., \& van den Brink, W. (2005). Decision making in pathological gambling: A comparison between pathological gamblers, alcohol dependents, persons with Tourette syndrome, and normal controls. Brain Research: Cognitive Brain Research, 23, 137-151. doi: 10.1016/j.cogbrainres.2005.01.017.

Gupta, R., \& Deverensky, J. L. (1998). Adolescent gambling behavior: A prevalence study and examination of the correlates associated with problem gambling. Journal of Gambling Studies, 14, 319-345.

Hagger, M. S., Wood, C., Stiff, C., \& Chatzisarantis, N. L. (2010). Ego depletion and the strength model of self-control: A meta-analysis. Psychological Bulletin, 136, 495-525. doi:10.1037/a0019486.

Johansson, A., Grant, J. E., Kim, S. W., Odlaug, B. L., \& Götestam, K. G. (2009). Risk factors for problematic gambling: A critical literature review. Journal of Gambling Studies, 25, 67-92. doi: 10.1007/s10899-008-9088-6.

Ladouceur, R., Arsenault, C., Dubé, D., Freeston, M. H., \& Jacques, C. (1997). Psychological characteristics of volunteers in studies on gambling. Journal of Gambling Studies, 13, 69-84.

Ladouceur, R., Boudreault, N., Jacques, C., \& Vitaro, F. (1999). Pathological gambling and related problems among adolescents. Journal of Child \& Adolescent Substance Abuse, 8, 55-68.

Muraven, M., Collins, R. L., Shiffman, S., \& Paty, J. A. (2005). Daily fluctuations in self-control demands and alcohol intake. Psychology of Addictive Behaviors, 19, 140-147. doi:10.1037/0893-164X.19.2.140.

Muraven, M., \& Slessareva, E. (2003). Mechanisms of self-control failure: Motivation and limited resources. Personality and Social Psychology Bulletin, 29, 894-906. doi:10.1177/0146167203253209.

Muraven, M., Tice, D. M., \& Baumeister, R. F. (1998). Self-control as limited resource: Regulatory depletion patterns. Journal of Personality and Social Psychology, 74, 774-789.

Neighbors, C., Lostutter, T. W., Cronce, J. M., \& Larimer, M. E. (2002). Exploring college student gambling motivation. Journal of Gambling Studies, 18, 361-370. doi:1050-5350/02/1200-0361/0.

Neri, D. F., Oyung, R. L., Colletti, L. M., Mallis, M. M., Tam, P. Y., \& Dinges, D. F. (2002). Controlled breaks as a fatigue countermeasure on the flight deck. Aviation, Space and Environmental Medicine, $73,654-664$.

Newby-Clark, I. R., \& Brown, A. (2008). Self-regulation and problem gambling behaviour. Final report on OPGRC level II grant. Ontario Problem Gambling Research Council (OPGRC).

Nower, L., Derevensky, J. L., \& Gupta, R. (2004). The relationship of impulsivity, sensation seeking, coping, and substance use in youth gamblers. Psychology of Addictive Behaviors, 18, 49-55. doi: 10.1037/0893-164X.18.1.49.

O'Connor, J., \& Dickerson, M. (2003). Impaired control over gambling in gaming machine and off-course gamblers. Addiction, 98, 53-60.

Shmueli, D., \& Prochaska, J. J. (2009). Resisting tempting foods and smoking behavior: Implications from a self-control theory perspective. Health Psychology, 28, 300-306. doi:10.1037/a0013826.

Thayer, R. E., Newman, J. R., \& McClain, T. M. (1994). Self-regulation of mood: Strategies for changing a bad mood, raising energy, and reducing tension. Journal of Personality and Social Psychology, 67, 910-925.

Tice, D. M., Baumeister, R. F., Shmueli, D., \& Muraven, M. (2007). Restoring the self: Positive affect helps improve self-regulation following ego depletion. Journal of Experimental Social Psychology, 43, 379-384. doi:10.1016/j.jesp.2006.05.007.

Tucker, P. (2003). The impact of rest breaks upon accident risk, fatigue and performance: A review. Work and Stress, 17, 123-137. doi:10.1080/0267837031000155949.

Tyler, J., \& Burns, K. (2008). After depletion: The replenishment of the self's regulatory resources. Self and Identity, 7, 305-321. doi:10.1080/15298860701799997.

Vohs, K. D., Baumeister, R. F., Schmeichel, B. J., Twenge, J. M., Nelson, N. M., \& Tice, D. M. (2008). Making choices impairs subsequent self-control: A limited-resource account of decision making, selfregulation, and active initiative. Journal of Personality and Social Psychology, 94, 883-898. doi: 10.1037/0022-3514.94.5.883.

Vohs, K. D., Finkenauer, C., \& Baumeister, R. F. (2011). The sum of friends' and lovers' self-control scores predicts relationship quality. Social Psychological and Personality Science, 2, 138-145. doi: $10.1177 / 1948550610385710$.

Vohs, K. D., \& Heatherton, T. F. (2000). Self-regulatory failure: A resource-depletion approach. Psychological Science, 11, 249-254.

Welte, J. W., Barnes, G. M., Tidwell, M. O., \& Hoffman, J. H. (2008). The prevalence of problem gambling among U.S. adolescents and young adults: Results from a national survey. Journal of Gambling Studies, 24, 119-133. 